\title{
Alianza terapéutica y su relación con las variables de apego del terapeuta y del consultante*
}

Therapist and Client Attachment and its Relationship with Therapeutic Alliance

Recibido: agosto 17 de 2010 | Revisado: diciembre 26 de 2010 | Aceptado: agosto 22 de 2011

\author{
CARMEN Elvira NAVIA ** \\ ERIKA MARgARITA ARIAS ***
}

Universidad Nacional de Colombia, Bogotá, Colombia

\section{RESU MEN}

El presente estudio se diseñó con el propósito de determinar las variaciones de la alianza terapéutica (AT) a lo largo de la terapia y determinar la relación con el apego del consultante y del terapeuta. El apego se evaluó mediante la Adult Attachment Scale y la AT a través del Inventario de Alianza Te-

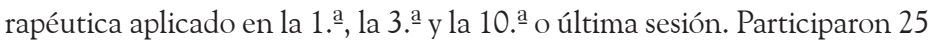
terapeutas, 19 estudiantes y 6 profesionales con 1 a 4 años de experiencia clínica. Se inició con 50 consultantes mayores de edad; para la 3. a sesión quedaron 30 casos y en la última, 15. Los resultados indicaron que la seguridad del apego favorece el desarrollo de la AT evaluada por el terapeuta, en especial en la 1. ㄹ sesión. Posteriormente, la apertura a la intimidad con el consultante y la confianza en el procedimiento terapéutico benefician la AT. Palabras clave autores:

Alianza terapéutica, apego.

Palabras clave descriptores:

Psicología clínica, psicoterapia, estudio longitudinal.

\section{A B S T R A C T}

This article reviews the results of a study on the development of the therapeutic alliance (TA) along the psychotherapeutic process and its relationship with therapists' and clients' attachment styles. TA was evaluated in the 1 st, 3rd, and 10th or last session through the "Inventario de Alianza Terapéutica" (IAT), attachment was measured by the Adult Attachment Scale. Twenty five therapists participated, 19 senior psychology students and 6 professional psychologists with 1 to 4 years of clinical experience. The study was initiated with 50 adult people, for the 3 rd session there were 30 cases, and for the last one, 15 . Secure attachment of the therapist and the client associated with better TA as evaluated by the therapist. Later on, intimacy and trustfulness in the therapeutic procedures improved the TA. Key words authors:

Therapeutic alliance, attachment.

Key words plus:

Clinical psychology, psychotherapy, longitudinal research. 
Desde finales del siglo pasado, la difusión de la medicina basada en la evidencia y la presión ejercida por aseguradoras y sistemas nacionales de salud (Beutler, 1998) hicieron cada vez más imperativa la necesidad de mostrar la efectividad, eficacia y eficiencia de los procedimientos e impusieron la aplicación de tratamientos empíricamente soportados (TES). Al igual que en otros países, la normatividad que reglamenta los servicios de salud en Colombia, incluidos entre ellos los centros de atención psicológica, indica que los prestadores de servicios de salud deben contar con "(...) guías clínicas de atención, preferiblemente de medicina basada en evidencia, para las patologías que constituyen las primeras 10 causas de consulta o egreso, o las 5 primeras causas para el caso de profesionales independientes (...)" (Ministerio de la Protección Social, 2006, p. 139). El auge de TES ha generado una amplia controversia alrededor de la importancia relativa de los factores relacionales y técnicos en la psicoterapia (Beutler \& Castonguay, 2006; Golfried \& Eubanks-Carter, 2004; Norcross, 2001; Wampold, 2001; Westen, Novotny \& Thompson-Brenner, 2004) y un renovado interés en el estudio de los factores comunes, en especial, lo relativo a las variables relacionales. $\mathrm{Mu}$ chas de las investigaciones sobre la contribución de los aspectos técnicos y comunes en el cambio indican que la varianza en los resultados terapéuticos es atribuible en mayor medida a los factores comunes (Beutler, 1989; Lambert, 1992; Wampold, 2001) y entre ellos, la alianza terapéutica (AT) es uno de los aspectos relacionales con mayor y más claro soporte empírico (Ackerman et al., 2001; Norcross, 2002). Según Horvath y Bedi (2002), aunque el tamaño del efecto en los estudios meta-analíticos sobre la relación entre AT y resultado terapéutico no es extremadamente alto (ES entre 0.21 y 0.25 ), la extensa revisión de estudios realizada por Wampold (2001) permite concluir que la AT, en conjunción con las variables del terapeuta, explica la mayor parte de la varianza en los resultados de la terapia. Así mismo, se ha encontrado que es uno de los predictores más robustos de resultados del tratamiento para adultos, niños y adolescentes (Hazell, 2003; Hovarth \& Bedi, 2002; Horvath \& Greenberg, 1989; Karver,
2003) y del involucramiento del consultante con la terapia (Karver et al., 2008).

La evidencia entonces apoya la importancia de la AT como factor de cambio terapéutico y resulta fundamental estudiar sistemáticamente su desarrollo y variabilidad a lo largo del tratamiento. DeRubeis, Brotman y Gibbons (2005) y Horvath y Bedi (2002) agrupan en cuatro categorías los factores moderadores y mediadores de la relación entre la AT y el resultado: 1) el consultante, 2) el terapeuta, 3) la interacción consultante-terapeuta y 4) las variables intraterapia, tales como la variación en la calidad de la AT a lo largo de las fases del tratamiento, la hipótesis sobre la relación de mutua retroalimentación entre AT y resultado y, por último, la posición de quien hace la observación. Respecto al último punto, Horvath y Bedi (2002) señalan que la AT valorada por los consultantes tiende a ser un mejor predictor de los resultados terapéuticos que la AT evaluada por el terapeuta; sin embargo, investigaciones recientes indican que las evaluaciones de la AT por parte de consultantes y terapeutas tienden a asemejarse a medida que la terapia avanza, y la evaluación de la AT por parte del terapeuta se convierte en un mejor predictor del resultado.

Aunque existen diferencias importantes entre los autores respecto al significado exacto del término AT, los distintos planteamientos confluyen en reconocer que esta implica una conexión emocional, es decir, de apego con el terapeuta, y una cognitiva, o de esperanza en el tratamiento y disposición para involucrarse en el mismo (Bordin, 1979; Karver, Handelsman, Fields \& Bickman, 2006). El vínculo de colaboración mutua, confianza, cuidado y respeto es uno de los aspectos fundamentales de la AT y eje fundamental para la exploración del mundo interno (Luborsky, 1984; Orlinsky, Grawe \& Parks, 1994; Parish \& Eagle, 2003; Safran \& Murran, 2000).

Las investigaciones sobre apego y relaciones adultas indican que las relaciones tempranas se convierten en una base para el desarrollo de relaciones adultas (Mallinckrodt, 1991), en especial aquellas que implican intimidad emocional (Collins \& Reed, 1990). En tanto que los terapeutas 
constituyen figuras fundamentales de apego para los consultantes (Bowlby, 1988; Parish \& Eagle, 2003), en años recientes se observa un creciente interés por estudiar el impacto de las experiencias relacionales previas, en especial del consultante, en el desarrollo de la alianza.

Los estudios apoyan, al menos en parte, la hipótesis de que el vínculo seguro del consultante favorece el desarrollo de la AT (Eames \& Roth, 2000; Goldman \& Anderson, 2007; Kanninen, Salo \& Punamäki, 2000; Meyer \& Pilkonis, 2001; Satterfield \& Lyddon, 1998; Strauss, 2000) mientras que el apego temeroso se asocia con puntuaciones más bajas (Eames \& Roth, 2000; Kanninen et al., 2000; Satterfield \& Lyddon, 1998; Strauss, 2000). Aunque algunos trabajos muestran una correlación significativa entre el estilo de apego del consultante y la AT en las primeras sesiones (Eames \& Roth, 2000), la mayoría de las investigaciones indica que esta relación comienza a ser significativa entre la 3. - y la 5. - sesión, y cada estilo de apego del consultante mostrará un desarrollo diferente de la AT a lo largo de la terapia. Al respecto, Kanninen et al. (2000) concluyen que los consultantes con apego seguro desarrollan una AT estable a lo largo del tratamiento, quienes manifiestan apego preocupado inician con bajos índices en la AT pero al final del tratamiento la evalúan muy positivamente, mientras que las personas con apego indiferente muestran deterioro en la AT hacia el final de la terapia. Además de las variaciones a lo largo del tratamiento, Kivlighan, Patton y Foote (1998) encontraron que el impacto del apego del consultante sobre la alianza terapéutica es moderado por la experiencia del terapeuta. Los terapeutas con mayor experiencia desarrollan mejores alianzas con los consultantes que presentan dificultades a nivel de cercanía y confianza, mientras que la experiencia no es una variable significativa con consultantes que no presentan dificultades vinculares.

Mientras que el estilo de apego del consultante ha recibido considerable atención, hay pocos estudios en relación con el impacto del estilo de apego del terapeuta (Strauss, 2000) y la evidencia al respecto indica que la seguridad del terapeuta se asocia con la habilidad para responder a las necesidades del consultante (Dozier, Cue \& Barnett, 1994) y con menores choques entre el terapeuta y sus consultantes (Mallinckrodt, 2000). Por el contrario, los terapeutas más ansiosos tienden a responder de manera menos empática ante las rupturas en la AT, en especial con consultantes ansiosos o seguros (Rubino, Barker, Roth \& Fearon, 2000) y el vínculo ansioso del terapeuta se asocia con problemas en el desarrollo de la AT temprana (Sauer, López \& Gormley, 2003).

En resumen, la AT se construye y varía a lo largo de la terapia y como proceso interpersonal, depende no solo de las destrezas técnicas del terapeuta, sino de sus características personales y de variables relacionales del consultante. En virtud de lo anterior y con el propósito de estudiar el papel de uno de los aspectos relacionales que caracterizan al terapeuta y al consultante al ingresar a la terapia y que pueden afectar el desarrollo de la AT, el presente trabajo buscó: 1) describir las variaciones en la evaluación de la AT por parte de terapeutas y consultantes a lo largo de las sesiones; 2) determinar si existía una relación entre las variables de apego del terapeuta y del consultante con la evaluación de la AT por parte del terapeuta y del consultante luego de la 1. . $^{\text {, }}$ la 3. y la 10. o o última sesión y 3) establecer si el apego del consultante y el del terapeuta predicen la evaluación de la AT en la 1. $\cdot$, la 3. - y la 10. última sesión.

\section{Método}

\section{Participantes}

Participaron 25 terapeutas, 19 estudiantes en formación que realizaban el año de práctica profesional para optar al título de pregrado en Psicología y 6 profesionales con 1 a 4 años de experiencia clínica posterior al grado; $51.72 \%$ de los estudiantes fueron mujeres y $48.27 \%$ hombres, con una edad promedio de 22 años (rango $=20$ a 28 años) y de los profesionales, el $45.45 \%$ fueron mujeres y el $54.54 \%$ hombres, con una edad promedio de 25.4 años (rango $=23$ a 29 años).

Siguiendo un instructivo elaborado por el equipo de investigación, los terapeutas a cargo de los 
casos le presentaban la investigación a cada nuevo consultante adulto que era admitido al Servicio de Atención Psicológica de la Universidad Nacional de Colombia (SAP); si el consultante aceptaba participar bajo las condiciones estipuladas, se firmaba el consentimiento informado. Del total de consultantes mayores de edad atendidos en terapia individual en el SAP entre enero de 2009 y abril de 2010, se obtuvieron datos completos para 50 personas que representan el $23.58 \%$ de los adultos admitidos durante este período; $60 \%$ fueron mujeres y $40 \%$ hombres, con edades comprendidas entre los $18 \mathrm{y}$ 58 años de edad $(M=29)$. El 69.5\% tenía formación universitaria o de posgrado, el 11.1\% formación universitaria incompleta y el $13.5 \%$ bachillerato; la mayoría provenían de estrato socioeconómico medio bajo (84.7\%) y fueron diagnosticados con trastornos adaptativos (33\%), seguido por trastornos de ansiedad (23\%), problemas de relación (20.8\%), trastornos del estado de ánimo (18.8\%) y trastornos de personalidad (4.2\%).

Para la tercera sesión solo se obtuvieron datos completos de 30 consultantes; se excluyeron 11 casos que tenían datos incompletos y 9 personas desertaron de la terapia. En la 10.․ㅗ sesión se contó con 15 casos, pues cinco desertaron de la terapia y 10 tenían datos incompletos.

\section{Variables e instrumentos}

\section{Alianza Terapéutica}

Luego de una extensa revisión sobre el concepto de AT en diferentes escuelas terapéuticas, Bordin (1979) desarrolló una definición comprehensiva que incluía los aspectos contemplados por las distintas orientaciones teóricas. De acuerdo con esto, en el presente trabajo se entendió la alianza como el aspecto propositivo y consiente de la relación que implica la colaboración activa entre los participantes a través de: 1) acuerdos sobre metas terapéuticas; 2) consenso sobre "tareas" o actividades terapéuticas y 3) el vínculo entre terapeuta y consultante. Para evaluarla se empleó el Inventario de Alianza Terapéutica (IAT) en la versión corta para el terapeuta y para el consultante, versión validada en es- pañol del Working Alliance Inventory (WAI), una medida ampliamente utilizada en la investigación sobre AT y desarrollada por Horvath y Greenberg (1989) con base en el modelo planteado por Bordin. La versión corta validada al español (Santibáñez, 2003) es una medida de autorreporte que consta de 12 ítems puntuados en una escala tipo Likert de 5 puntos en la que un mayor puntaje indica una mejor evaluación de AT. Las propiedades psicométricas del IAT muestran una adecuada confiabilidad con valores de 0.67 para la escala de vínculo, 0.8 en tareas, 0.78 para las metas y 0.9 para la escala total en la versión del consultante. En la versión del terapeuta, la confiabilidad fue de 0.7 para la escala vínculo, 0.85 en tareas, 0.88 en metas y 0.93 en la escala total (Santibáñez, 2003).

\section{Apego Adulto}

Para evaluar el apego se empleó la Escala de Apego Adulto (Adult Attachment Scale- AAS) desarrollada por Collins y Read (1990) a partir de la categorización del apego adulto que hicieron Hazan y Sharver (1987). Para estos autores, los adultos presentan diferentes estilos de apego que se construyen a partir de experiencias relacionales con personas significativas en su vida (relaciones con los padres, relaciones de pareja, etc.). Los diferentes estilos de apego están relacionados directamente con las creencias que se tienen de uno mismo y acerca de las relaciones sociales. A diferencia de otros instrumentos diseñados para evaluar el apego adulto, la AAS ofrece una medida en las dimensiones fundamentales del apego, al tiempo que permite establecer categorías.

La AAS evalúa tres dimensiones de apego: 1) dependencia o posibilidad de confiar en otros; 2) ansiedad o temor al abandono y 3) proximidad o comodidad con las relaciones íntimas. Consta de 18 ítems calificados en una escala tipo Likert de cinco puntos en la que un mayor puntaje indica una mayor presencia de la dimensión evaluada.

Se realizó un procedimiento de adaptación de la escala mediante la traducción de los reactivos y posterior validación por jueces expertos indicando: 1) posibles efectos de diferencias culturales; 2 ) vali- 
dez del constructo para la población de Bogotá y sus alrededores; 3) pertinencia del lenguaje empleado en la redacción de los ítems y 4) equivalencia con la escala original (International Test Commission, 2010). El procedimiento de jueces expertos fue realizado por dos psicólogas profesionales, profesoras universitarias con dominio del idioma inglés y con conocimiento de las teorías de apego. Posteriormente, se aplicó a una muestra de 370 personas, 157 hombres y 208 mujeres entre 16 y 75 años de edad y se aplicaron los mismos análisis psicométricos del estudio original (Collins \& Read, 1990). Los resultados indicaron que para la muestra de estandarización la prueba AAS tiene una confiabilidad media $(\alpha=0.52)$ y muestra una estructura factorial diferente a la de la escala original (Navia \& Arias, 2010), razón por la cual se recomendó ser cautelosos con el nivel de generalización de los resultados a partir de ella.

\section{Procedimiento}

Tanto a los terapeutas profesionales como a cada grupo nuevo de estudiantes que ingresaba a la práctica en el SAP se les informó sobre la investigación y la importancia de la misma, haciendo claridad en que su participación no tendría repercusiones contractuales para los profesionales ni en la evaluación de la práctica para los estudiantes. Los terapeutas que decidieron participar firmaron un consentimiento informado y respondieron la AAS. Antes de iniciar la primera sesión, el terapeuta informaba a cada consultante mayor de edad sobre la investigación y le solicitaba su participación. Los consultantes que de manera libre y voluntaria decidían hacerlo debían firmar un consentimiento informado y se procedía a grabar la sesión. Al finalizar respondían la AAS y tanto el terapeuta como el consultante evaluaban la alianza mediante las respectivas formas del IAT. La aplicación del IAT se repetía en la 3. - y en la 10. o última sesión del tratamiento.

\section{Resultados}

Se inició con un análisis descriptivo de los puntajes de apego de los participantes y de la AT en las tres sesiones evaluadas. Posteriormente, y con ayuda del software SPSS 11.0, se realizaron análisis correlacionales y de regresión lineal múltiple para las sesiones 1,3 y 10 con todas las variables de interés del estudio. Para los análisis de regresión, se tuvieron en cuenta las variables de apego del terapeuta y el consultante, así como las puntuaciones obtenidas en el IAT. Previo cumplimiento de los supuestos de homocedasticidad se empleó el método de estimación por pasos sucesivos, puesto que no se sabía a ciencia cierta cuáles de las múltiples variables podían predecir la puntuación en el IAT. Para la 10.a sesión, y dado el pequeño número de observaciones, se optó por realizar un análisis de correlación no paramétrico con el fin de controlar el error tipo I debido a falta de homocedasticidad.

\section{Análisis de las medidas de alianza y apego}

En relación con los puntajes obtenidos por la muestra colombiana con la que se hizo la estandarización, las puntuaciones obtenidas por los consultantes en la dimensión de ansiedad de la AAS fueron más altas, mientras que los puntajes en proximidad y cercanía fueron similares. En las dimensiones de proximidad y dependencia los terapeutas tuvieron puntuaciones mayores que los consultantes, no así en la dimensión de ansiedad. Como se observa en la Tabla 1, excepto en la dimensión de ansiedad, las puntuaciones obtenidas por los consultantes en la AAS presentaron una mayor variabilidad y al compararlas con la muestra de terapeutas se encontraron diferencias estadísticamente significativas con un $95 \%$ de confianza en dependencia $(t=2.75, p=0.008)$, ansiedad $(t=5.15, p=0.000)$ y proximidad $(t=4.038, p=0.000)$.

Como se observa en la Figura 1, la puntuación total obtenida en la escala de AT mostró un incremento a lo largo de las sesiones, en especial entre la 1..$^{\mathrm{a}}$ y la 3.a (Figura 1).

La dimensión vincular de la AT fue la que obtuvo puntajes más altos a lo largo de las tres sesiones, tanto por parte de los consultantes como de los terapeutas (Tabla 2), y no se encontraron diferencias estadísticamente significativas al comparar las puntuaciones obtenidas por ambos grupos. 
TABLA 1

Estadísticos descriptivos de las puntuaciones obtenidas por consultantes y terapeutas en las dimensiones de la AAS

\begin{tabular}{lcccccc}
\hline & \multicolumn{2}{c}{ Dependencia } & \multicolumn{2}{c}{ Ansiedad } & \multicolumn{2}{c}{ Proximidad } \\
& Terapeuta & Consultante & Terapeuta & Consultante & Terapeuta & Consultante \\
\hline Media & 19.7 & 17.5 & 12.3 & 17.2 & 22.8 & 19.4 \\
DE & 3.9 & 4.3 & 5.4 & 4.3 & 3 & 4.6 \\
Mínimo & 10 & 7 & 6 & 9 & 18 & 8 \\
Máximo & 26 & 29 & 23 & 28 & 29 & 29 \\
\hline
\end{tabular}

Fuente: elaboración propia.

TABLA 2

Puntuaciones de AT a través de las diferentes sesiones

\begin{tabular}{lcccccc}
\hline & \multicolumn{2}{c}{ Sesión 1 } & \multicolumn{2}{c}{ Sesión 3 } & \multicolumn{2}{c}{ Sesión 10 } \\
\multicolumn{1}{c}{ Promedio } & Consultante & Terapeuta & Consultante & Terapeuta & Consultante & Terapeuta \\
\hline Total & 44.3 & 44.9 & 48.9 & 49.3 & 50 & 49.1 \\
Vínculo & 15.5 & 17.5 & 17.3 & 18.5 & 17.3 & 17.8 \\
Metas & 14 & 13.4 & 15.5 & 15.1 & 16.1 & 15.3 \\
Tareas & 14.8 & 14.1 & 16.1 & 15.7 & 16.6 & 16 \\
\hline
\end{tabular}

Fuente: elaboración propia.

Análisis de relaciones entre variables de apego del terapeuta y del consultante con la AT

Los análisis para la 1.로 sesión indicaron una correlación negativa de magnitud baja, pero estadísticamente significativa, entre la puntuación de alianza total evaluada por el terapeuta y sus puntuaciones en la dimensión de ansiedad $(r=-0.281, p=0.048)$; una correlación moderadamente positiva entre la dimensión de dependencia del consultante y la evaluación del terapeuta en la dimensión del vínculo del IAT $(r=0.314, p=0.027)$. En el caso de la AT evaluada por el consultante no se encontraron relaciones significativas con ninguna de las dimensiones de apego.

En la 3.a sesión, nuevamente se encontró correlación moderada y estadísticamente significativa entre la dimensión de dependencia del consultante y el vínculo evaluado por el terapeuta en el IAT $(r=0.369, p=0.041)$ y aparece una nueva relación entre la dimensión de proximidad del terapeuta y la de tareas de la AT evaluada por el terapeuta $(r=0.369$, $p=0.041)$. A diferencia de la 1. $\cdot$ a sesión, en esta sí se encuentran relaciones entre el apego y la AT evaluada por el consultante, específicamente, entre el vínculo y las puntuaciones del consultante en dependencia $(r=0.437, p=0.012)$ y ansiedad $(r=-0.41, p=0.02)$. Finalmente, durante la 10. ${ }^{a}$ sesión las únicas variables que mostraron asociación significativa fueron la de ansiedad del terapeuta con la dimensión de tareas evaluada por el consultante $(r=-0.468, p=0.033)$ y la de dependencia del consultante con el vínculo percibido por el mismo $(r=0.453, p=0.039)$.

En su investigación sobre el apego, la calidad de las relaciones de objeto y la AT, Goldman y Anderson (2007) plantean que resulta más útil evaluar el apego en una variable bipolar continua entre inseguridad y seguridad. Para calcularla usan la fórmula propuesta por Allen, Huntoon y Evans (1999), según la cual el puntaje de apego seguro se obtiene sumando las dimensiones de proximidad y dependencia, y restando la escala de ansiedad.

$\mathrm{Al}$ trabajar el apego en términos de esta dimensión, se encontraron correlaciones moderadas y 


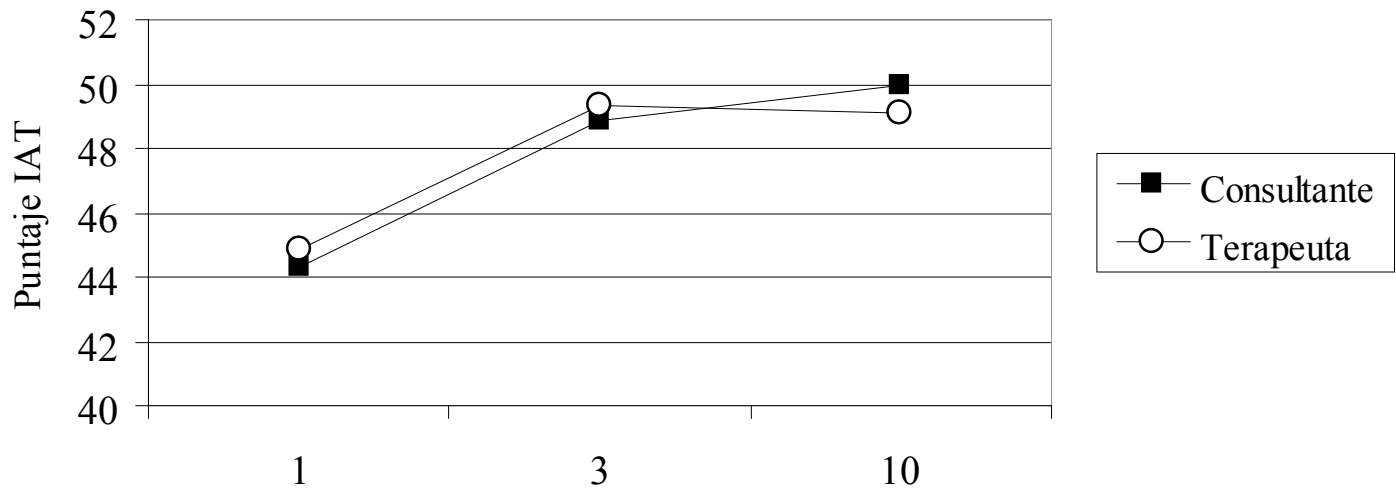

Sesiones

Figura 1. Puntuaciones de la AT total a través de las sesiones. Fuente: elaboración propia.

estadísticamente significativas entre el apego seguro del terapeuta y la puntuación total del IAT respondido por el terapeuta en la 1. ․ $^{\mathrm{a}}$ sesión $(r=0.359$, $p=0.11)$, particularmente entre las dimensiones de tareas $(r=0.334, p=0.018)$ y metas $(r=0.29$, $p=0.041)$. También se encontró asociación positiva y significativa entre el apego seguro del consultante y la puntuación de alianza total en la 1.ㄹ sesión según la evaluación del terapeuta. Los análisis de correlación para las sesiones 3 y 10 mostraron asociación moderada pero significativa entre las variables de apego seguro del terapeuta y el vínculo percibido por el terapeuta $(r=0.381, p=0.035)$.

Los análisis de regresión indicaron que para la 1.a sesión la puntuación de alianza percibida por el terapeuta puede ser predicha en un $13 \%$ por el nivel de apego seguro del consultante $(\beta=0.35$, $p=0.011)$, mientras que el modelo que incluye las variables de apego del terapeuta y del consultante no resultó predictivo de la puntuación de alianza total percibida por el consultante. Para la 3 a a sesión, un modelo compuesto por la dimensión de proximidad del terapeuta $(\beta=0.439, p=0.035)$ y del consultante $(\beta=0.458, p=0.032)$ puede explicar el 33\% de la varianza de la puntuación de AT percibida por el terapeuta; por el contrario, para la alianza percibida por el consultante ningún modelo resultó significativo.

\section{Discusión}

Los resultados de este estudio indican que la relación entre el apego y la AT es compleja, variará en función de quien evalúe y del momento del proceso en el que se realice la medición.

Como lo han mostrado investigaciones anteriores, es a partir de la 3. a sesión que, desde la perspectiva del consultante, las variables de apego comienzan a jugar un papel en el desarrollo de la AT (Kanninen et al., 2000). Una vez expuesto el problema y el motivo por el cual se acude en busca de ayuda, comienza una exploración más profunda e íntima de la persona, su historia y su situación vital, que puede resultar angustiosa; de allí que en este momento resultan fundamentales para el consultante su confianza en el terapeuta y la seguridad en la permanencia del vínculo, factores estos que favorecerán su compromiso con la terapia.

Desde la perspectiva de los terapeutas, los resultados muestran que estos son más susceptibles que los consultantes a las variables relacionadas con el apego, en especial en la primera sesión. Como lo indican estudios anteriores (Dozier et al., 1994; Rubino et al., 2000; Sauer et al., 2003), la ansiedad del terapeuta, su temor a ser abandonado y la falta de seguridad y confianza en sí mismo afectan su capacidad para desarrollar la relación empática y 
segura que es fundamental en las primeras fases de la terapia (Luborsky, 1994). Al mismo tiempo, el apego seguro del consultante permite que el terapeuta se sienta cómodo y confiado para dar apoyo y soporte y, por lo tanto, desarrollar una mejor AT. Como lo han concluido algunos autores (Kivlighan et al., 1998), los terapeutas con menor experiencia, como es el caso de quienes participaron en el estudio, desarrollan mejor la AT con consultantes que presentan apego seguro. Por otro lado, pareciera que la confianza en sí mismos y el buen nivel de estima personal que caracteriza a las personas con apego seguro, permite que los terapeutas que muestran altos puntajes en esta dimensión puedan plantear con mayor claridad y asertividad las metas de la terapia y los procedimientos mediante los que se alcanzarán.

Luego del alivio logrado tras una escucha atenta en las primeras sesiones, terapeuta y consultante deberán definir y comprometerse con lo que se busca lograr a través de la terapia y los medios que emplearán para ello. Según Luborsky (1994), la fe del consultante en los procedimientos y su compromiso con lo que la terapia demanda de él o ella, juegan en ese momento del proceso un papel fundamental en definir la AT. En el presente trabajo, lo anterior parece aplicarse más a los terapeutas que a los consultantes; su disponibilidad para desarrollar relaciones próximas e íntimas y su confianza y compromiso con los procedimientos escogidos, fueron los aspectos del apego que, desde la perspectiva de los terapeutas, asociaron con una mejor AT en la dimensión de tareas.

Se puede concluir que, al igual que para los terapeutas con mayor experiencia y formación, para quienes apenas inician su proceso de entrenamiento las variables de apego, tanto propias como del consultante, son factores importantes en el desarrollo de la AT y sería aconsejable tenerlas en cuenta como parte de los programas de formación. El primer encuentro con el consultante despierta el temor al abandono de los terapeutas pues los expone ante la posibilidad de lograr comprometer al consultante o perder la posibilidad de trabajar con él o ella; de allí que la ansiedad frente al abandono sea un factor importante en el desarrollo de la primera sesión, y los terapeutas en formación deberán aprender a manejarla. Así mismo, a medida que avanza la terapia es necesario desarrollar con los terapeutas competencias para establecer relaciones íntimas y cercanas que permitan la reflexión profunda, así como promover su confianza en los procedimientos y convencimiento sobre su efectividad.

Si bien los análisis mostraron correlaciones significativas, es necesario mantener en mente que fueron moderadas y esto puede atribuirse, en parte, a la confiabilidad moderada del instrumento y, por otro lado, al efecto de otras variables como la edad y género de los participantes, el diagnóstico, la orientación teórica, entre otras, que pueden afectar la AT. Así mismo, es necesario ser cuidadosos al generalizar los resultados pues la muestra se limita a terapeutas con formación de pregrado que trabajan con una población que no presenta graves perturbaciones psicológicas ni serias lesiones a nivel vincular. En este sentido, se trata de consultantes capaces de desarrollar relaciones más o menos estables e íntimas. Además, la muestra no fue aleatoria y quienes decidieron participar lo hicieron porque se sentían tranquilos de ser grabados y evaluados con fines diferentes a los de la terapia, situación que ya indica cierto nivel de seguridad en sí mismos y en otros. Es necesario también anotar que para facilitar la recolección de los datos fueron los terapeutas mismos quienes entregaron y recibieron los cuestionarios IAT de los consultantes, factor que pudo influir en sus respuestas.

A pesar de las limitaciones, el estudio evidencia la necesidad de tener en cuenta los aspectos personales del terapeuta en el proceso de formación y dado que la AT es de fundamental importancia para el desarrollo de la terapia, es recomendable continuar determinando las variables que afectan su desarrollo.

\section{Referencias}

Ackerman, S. J., Smith Benjamin, L, Beutler, L. E., Gelso, C. J., Golfried, M. R., Hill, C., et al. (2001). Empirically supported therapy relationships: Conclusions and recommendations of the Division 29 task force. Psychotherapy, 38(4), 495-497. 
Allen, J. G., Huntoon, J. \& Evans, R. B. (1999). Complexities in complex posttraumatic stress disorder in inpatient women: Evidence from cluster analysis of MCMI-II personality disorder scales. Journal of Personality Assessment, 73, 449-471.

Beutler, L. E. (1989). Differential treatment selection: The role of diagnosis in psychotherapy. Psychotherapy, 26, 271-281.

Beutler, L. (1998). Identifying empirically supported treatments. iWhat if we didn't? Journal of Consulting and Clinical Psychology, 66(1), 113-120.

Beutler, L. E. \& Castonguay, L. G. (2006). The task force on empirically based principles of therapeutic change. En L. G. Castonguay \& L. E. Beutler (2006). Principles of therapeutic change that work (pp. 3-10). New York: Oxford University Press.

Bordin, E. (1979). The generalizability of the psychoanalytic concept of the working alliance. Psychotherapy: Theory, Research and Practice, 16(3), 252-260.

Bowlby, J. (1988). A secure base: Clinical applications of attachment theory. London: Routledge \& Kegan Paul.

Collins, N. L. \& Read, S. J. (1990). Adult attachment, working models, and relationship quality in dating couples. Journal of Personality and Social Psychology, 58, 644-663.

DeRubeis, R. J., Brotman, M. A. \& Gibbons, C. J. (2005). A conceptual and methodological analysis of the nonspecific argument. Clinical Psychology: Science and Practice, 12, 174-183.

Dozier, M., Cue, K. \& Barnett, L. (1994). Clinicians as caregivers: The role of attachment organization in treatment. Journal of Consulting and Clinical Psychology, 62, 793-800.

Eames, V. \& Roth, A. (2000). Patient attachment orientation and the early working alliance: A study of patient and therapist reports of alliance quality and ruptures. Psychotherapy Research, 10(4), 421-434.

Goldman, G. \& Anderson, T. (2007). Quality of object relations and security of attachment as predictors of early therapeutic alliance. Journal of Counseling Psychology, 54(2), 111-117.

Golfried, M. R. \& Eubanks-Carter, C. (2004). On the need for a new psychotherapy research paradigm: Comment on Westen, Novotny, and Thompson-Brenner (2004). Psychological Bulletin, 130(4), 669-673.
Hazan, C. \& Shaver, P. (1987). Romantic love conceptualized as an attachment process. Journal of Personality and Social Psychology, 52, 511-524.

Hazell, P. (2003). Review: Therapeutic relationship is modestly correlated with treatment outcome in child and adolescent psychotherapy. EvidenceBased Mental Health, 6(4), 122.

Horvath, A. O. \& Bedi, R. P. (2002). The Alliance. En J. C. Norcross (Ed.), Psychotherapy relations that work (pp. 37-69). Oxford: University Press.

Horvath, A. O. \& Greenberg, L. S. (1989). Development and validation of the working alliance inventory. Journal of Counseling Psychology, 36, 223-233.

International Test Commission. (2010). International Test Commission Guidelines for Translating and Adapting Tests. Consultado en febrero de 2011, en http://www.intestcom.org

Kanninen, K., Salo, J. \& Punamaki, R. -L. (2000). Attachment patterns and working alliance in trauma therapy for victims of political violence. Psychotherapy Research, 10(4), 435-449.

Karver, S. (2003). Prediction of treatment outcome from relationship variables in child and adolescent therapy: A meta-analytic review. Journal of Consulting and Clinical Psychology, 71, 452-464.

Karver, M. S., Handelsman, J. B., Fields, S. \& Bickman, L. (2006). Meta-analysis of therapeutic relationship variables in youth and family therapy: The evidence for different relationship variables in the child and adolescent treatment outcome literature. Clinical Psychology Review, 26, 50-65.

Karver, M., Shirk, S., Handelsman, J., Fields, S., Crisp, H., Gudmunsen, G. et al., (2008). Relationships processes in youth psychotherapy: Measuring alliance, alliance-building behaviors, and client involvement. Journal of Emotional and Behavioral Disorders, 16(15), 15-28.

Kivlighan, D. M., Jr., Patton, M. J. \& Foote, D. (1998). Moderating effects of client attachment on the counselor experience-working alliance relationship. Journal of Counseling Psychology, 45(3), 274-278.

Lambert, M. J. (1992). Psychotherapy outcome research: Implications for integrative and eclectic theories. En J. C. Norcross \& M. R. Goldfried (Eds.), Handbook of psychotherapy integration (pp. 94-129). New York: Basic Books, Inc. 
Luborsky, L. (1984). Principles of psychodynamic therapy. A manual for supportive-expressive treatment. New York: Basic Books, Inc.

Luborsky, L. (1994). Therapeutic alliance as predictors psychotherapy outcomes: Factors explaining the predictive success. En A. O. Horvath \& L. S. Greenberg (Eds.), The working alliance. Theory, research, and practice (pp. 38-51). New York: John Wiley \& Sons.

Mallinckrodt, B. (1991). Clients' representations of childhood emotional bonds with parents, social support, and formation of the working alliance. Journal of Counseling Psychology, 38(4), 401-409.

Mallinckrodt, B. (2000). Attachment, social competencies, social support, and interpersonal process in psychotherapy. Psychotherapy Research, 10(3), 239-266.

Meyer, B. \& Pilkonis, P. P. (2001). Attachment style. Psychotherapy, 38(4), 466-472.

Ministerio de la Protección Social. (2006). Anexo Técnico No. 1 de la Resolución 1043, 2006. Consultado en Enero de 2011, en http://www.minproteccionsocial.gov.co/salud/Paginas/SistemaObligato riodeGarant\%C3\%ADadeCalidad-SOGC.aspx

Navia, C. E. \& Arias, E. M. (2010, agosto). Personalidad, estilo comunicativo y apego del terapeuta y del consultante en relación con la calidad de la alianza terapéutica (Informe Técnico final de proyecto). Bogotá: Universidad Nacional de Colombia.

Norcross, J. (2001). Purposes, processes, and products of the Task Force on empirically supported therapy relationships. Psychological Bulletin, 38(4), 345-356.

Norcross, J. C. (Ed.). (2002). Psychotherapy relations that work. Oxford: University Press.

Orlinsky, D. E., Grawe, K. \& Parks, B. K. (1994). Process and outcome in psychotherapy-Noch Einmal. En
A. E. Bergin \& S. L. Garfield (Eds.), Handbook of psychotherapy and behavior change (pp. 270-379). New York: John Wiley \& Sons, Inc.

Parish, M. \& Eagle, M. (2003). Attachment to the therapist. Psychoanalytic Psychology, 20(2), 271-286.

Rubino, G., Barker, C., Roth, T. \& Fearon, P. (2000). Therapist empathy and depth of interpretation in response to potential alliance ruptures: The role of therapist and patient attachment styles. Psychotherapy Research, 10(4), 408-420.

Safran, J. D. \& Murran, J. C. (2000). Negotiating the therapeutic alliance. A relational treatment guide. New York: The Guildford Press.

Santibáñez, P. (2003). La alianza terapéutica en psicoterapia: el 'Inventario de Alianza de Trabajo' en Chile. Psykhe, 12(1), 109-118.

Satterfield, W. \& Lyddon, W. (1998). Client attachment and the working alliance. Counseling Psychology Quarterly, 11(4), 407-415.

Sauer, E. M., López, F. G. \& Gormley, B. (2003). Respective contributions of therapist and client adult attachment orientations to the development of the early working alliance: A preliminary growth modeling study. Psychotherapy Research, 13(3), 371-382.

Strauss, B. (2000). Attachment theory and psychotherapy research-Editor's introduction to a special section. Psychotherapy Research, 10(4), 381-389.

Wampold, B. E. (2001). The great psychotherapy debate: Models, methods, and findings. Mahwah, NJ: Erlbaum Associates.

Westen, D., Novotny, C. M. \& Thompson-Brenner, H. (2004). The empirical status of empirically supported psychotherapies: Assumptions, findings, and reporting in controlled clinical trials. Psychological Bulletin, 130(4), 631-663. 